\title{
Ketamin: Einsatz bei chronischen Schmerzen und Depression
}

\author{
Wolfgang Jaksch $(\mathbb{D} \cdot$ Rudolf Likar $\cdot$ Martin Aigner
}

Eingegangen: 12. Februar 2018 / Angenommen: 29. März 2019 / Online publiziert: 12. April 2019

(C) Der/die Autor(en) 2019

\begin{abstract}
Zusammenfassung Obwohl Ketamin seit langem bekannt und im klinischen Einsatz ist, bleiben nach wie vor Fragen rund um die vielfältigen möglichen Anwendungsfelder als Anästhetikum und Analgetikum offen. Diese betreffen nicht das klassische Einsatzgebiet in der Notfallmedizin und Anästhesie, sondern auch potentielle, neue Indikationen, in denen Ketamin in niedrigen, subanästhetischen Dosierungen zum Einsatz kommt. Der Wirkmechanismus am NMDA-Rezeptor unterscheidet Ketamin deutlich von allen anderen Analgetika. Mögliche Einsatzgebiete sind Prävention chronischer postoperativer Schmerzen sowie die Behandlung neuropathischer Schmerzen. Mit der Behandlung der refraktären Depression könnte sich Ketamin auch in einem gänzlich neuen Feld etablieren.
\end{abstract}

Schlüsselwörter Schmerzmedizin · S-Ketamin · Tumorschmerzen - Neuropathische Schmerzen · Therapieresistente Depression

Dr. W. Jaksch $(\bowtie) \cdot$ Prim. Univ.-Prof. Dr. R. Likar, MSc Prim. Ao.Univ.-Prof. Dr. M. Aigner

Österreichische Schmerzgesellschaft, Wien, Österreich wolfgang.jaksch@chello.at

Dr. W. Jaksch

Abteilung für Anästhesiologie, Intensiv- und

Schmerzmedizin, Wilhelminenspital der Stadt Wien,

Montlearstraße 37, 1160 Wien, Österreich

Prim. Univ.-Prof. Dr. R. Likar, MSc

Österreichische Gesellschaft für Anästhesiologie, Reanimation und Intensivmedizin, Wien, Österreich Abteilung für Anästhesiologie und Intensivmedizin, Klinikum Klagenfurt am Wörthersee, Klagenfurt am Wörthersee, Österreich

Prim. Ao.Univ.-Prof. Dr. M. Aigner

Abteilung Erwachsenenpsychiatrie, Universitätsklinikum Tulln, Tulln, Österreich

\section{Ketamine in the therapy of chronic pain and depression}

Summary Although ketamine has been known and clinically applied for a long time, questions still arise around the many possible indications in which the anesthetic and analgesic substance could be used. In particular, these questions relate to new indications in which ketamine is used in low subanesthetic doses. The mechanism of action at the NMDA receptor clearly distinguishes ketamine from all other analgesics. Possible applications include the prevention of chronic postoperative pain as well as the treatment of neuropathic pain. With the treatment of refractory depression completely new therapeutic areas for ketamine could be established.

Keywords Pain therapy - S-Ketamine - Cancer pain · Neuropathic pain · Therapy resistant depression

\section{Einleitung}

Das in den 1960er Jahren entwickelte Anästhetikum und Analgetikum Ketamin wurde über 50 Jahre vorwiegend in der Unfall- und Notfallmedizin eingesetzt, da es rasche Schmerzlinderung bis hin zur tiefen Anästhesie ohne die Gefahr einer Atemdepression erlaubt. Mittlerweile steigt jedoch auch das Interesse an Ketamin in niedrigen, nicht-anästhetischen Dosierungen. Potentielle Indikationen sind hier der perioperative Einsatz zur Vorbeugung von Hyperalgesie und chronischen postoperativen Schmerzen sowie die Behandlung chronischer Schmerzen [1, 4] und therapieresistenter Depressionen [2, 4]. Wie sich die Evidenzlage bzw. Kasuistiken für Ketamin in diesen Indikationsbereichen darstellen, und welche Behandlungsempfehlungen daraus abgeleitet werden können, ist Gegenstand der nachfolgenden Übersichtsarbeit. 


\section{Eigenschaften von Ketamin}

Ketamin ist ein chirales Derivat von Cyclohexanon mit einem Stereozentrum und gehört zur Familie der Arylcyclohexylamine. Damit ist es strukturell verwandt mit zahlreichen weiteren Anästhetika und psychoaktiven Substanzen wie Methoxetamin (MXE) oder Phencyclidin (PCP). Ketamin wurde 1962 eingeführt und gilt aufgrund der Kombination von psychotroper und schmerzstillender Wirkung als „dissoziatives Anästhetikum“ [1-3].

\section{Pharmakokinetik und Anwendungsformen}

Die Metabolisierung von Ketamin erfolgt in der Leber über das Cytochrome P-450 System, vermutlich über CYP 3A und CYP 3A 4, CYP 2B6 und CYP 2C9, die Ausscheidung über die Niere ist minimal. Allerdings sind hier noch nicht alle Fragen geklärt. Denn während die Inhibition von CYP 3A beispielsweise durch Clarithromycin oder Grapefruitsaft nach Einnahme von oralem S-Ketamin die Exposition erhöht, dürfte der CYP 3A4 Inhibitor Itraconazol nicht mit S-Ketamin interagieren. Die potenten CYP 3A Induktoren Rifampicin und Johanneskraut senken die Plasma-Spiegel von S-Ketamin nach oraler Einnahme sehr deutlich, nach intravenöser Applikation jedoch sehr viel geringer.

Die Metabolite sind pharmakologisch aktiv, Norketamin (Metabolite I) wurde bis vor kurzem als der wichtigste Metabolit betrachtet. Die Bedeutung von Norketamin wird allerdings gegenwärtig in Zweifel gezogen. Zwar wird Ketamin relativ rasch zu Norketamin N-demethyliert, doch dürfte Norketamin selbst rascher zu 6-Hydroxynorketamin weitermetabolisiert werden als bis vor kurzem angenommen und nicht den wichtigsten Metaboliten im Plasma darstellen. Norketamin weist ungefähr ein Drittel der anästhetischen Wirksamkeit von Ketamin auf. Die Halbwertszeit von Ketamin in der Beta-Phase beträgt in etwa 2,5 bis $3 \mathrm{~h}$, die Geschwindigkeit der Ausscheidung dürfte vom Blutfluss in der Leber abhängen [1, 3].

\section{Intravenöse Injektion/Infusion}

Sowohl racemisches Ketamin als auch S-Ketamin sind in Verwendung. Ein rezenter Review weist allerdings darauf hin, dass S-Ketamin besser titrierbar sein dürfte, da es eine höhere Clearance und einen steileren Anstieg des Effekts mit der Konzentration aufweist (concentration-effect curve) und das Fehlen des R-Enantiomers metabolische Interaktionen vermeidet. In der Anästhesie werden für S-Ketamin i.v. Induktionsdosen von $0,5-1,0 \mathrm{mg} / \mathrm{kg}$, gefolgt von wiederholten Erhaltungsdosen von $0,25-0,5 \mathrm{mg} / \mathrm{kg}$ (Bolus) oder kontinuierlicher Infusion von $0,5-3,0 \mathrm{mg} / \mathrm{kg} / \mathrm{h}$ empfohlen. Im analgetischen Einsatz liegen die BolusDosen bei $0,1-0,25 \mathrm{mg} / \mathrm{kg}$ gefolgt von Dauerinfusion mit $0,2-1,0 \mathrm{mg} / \mathrm{kg} / \mathrm{h}$. Beim Einsatz von racemischen Ketamin sind diese Dosierungen zu verdoppeln. Nach intravenöser Applikation von Ketamin kommt es zu einem initialen Anfluten (Alpha-Phase) mit einer Halbwertszeit von zehn bis 15 min und einer Gesamtdauer von ca. $45 \mathrm{~min}$ [3].

\section{Orale Einnahme}

Die Bioverfügbarkeit von oralem Ketamin ist aufgrund eines ausgeprägten First-Pass Metabolismus schlecht. Nur rund $17-24 \%$ von oralem racemischem Ketamin und $8-11 \%$ von oralem S-Ketamin erreichen die systemische Zirkulation. Aufgrund der Metabolisierung in der Leber gelangt nach oraler Einnahme von S-Ketamin sehr viel mehr S-Norketamin als Ketamin in die Zirkulation. Ein steady state von oralem S-Ketamin wird nach der dritten Dosis erreicht, es kommt zu keiner Akkumulation von S-Ketamin oder seinen Metaboliten, wenn S-Ketamin zweimal täglich oral eingenommen wird [5]. Da S-Norketamin ein schwächeres Analgetikum als S-Ketamin ist, wird geschätzt, dass bei oraler Einnahme im Vergleich zur intravenösen die Dosis um einen Faktor 2,4 erhöht werden muss, um die gleiche AUC (area under the curve) zu erreichen [3].

\section{Intramuskuläre Injektion}

Ketamin kann auch intramuskulär injiziert werden. Dabei kommt es zu rascher Absorption bei einer Bioverfügbarkeit von $93 \%$. Bereits nach vier Minuten ist Ketamin im Plasma nachweisbar, Peak-Konzentrationen werden nach fünf bis 30 min erreicht. Bereits ein bis fünf Minuten nach der Injektion setzt die Wirkung ein. Aufgrund des schnellen Wirkeintritts ist die i.m. Injektion eine Alternative zur intravenösen Applikation, wenn kein geeigneter intravenöser Zugang verfügbar ist [3].

\section{Sublinguale Applikation}

Sublinguale Formulierungen von Ketamin sind in Entwicklung als anwenderfreundliche Optionen für die Drittlinien-Therapie von Schmerzpatienten. Die Bioverfügbarkeit ist nicht besser als bei oraler Einnahme. Allerdings ist die Metabolisierung zu Norketamin weniger ausgeprägt und der maximale PlasmaSpiegel (Cmax) wird schneller erreicht (0,5 vs. $2 \mathrm{~h}$ ). Die sublinguale Administration kann vorteilhaft sein, wenn schnelle Wirkung angestrebt wird, was beim Akutschmerz oder bei Durchbruchschmerzen in der onkologischen Schmerztherapie der Fall sein kann [3, $6,7]$.

\section{Nasale Applikation}

Die intranasale Administration von Ketamin umgeht die First Pass Metabolisierung in der Leber. Damit bleibt auch die Metabolisierung zu Norketamin gering. Mit rund $45 \%$ ist die Bioverfügbarkeit von na- 
salem Ketamin etwas besser als bei oraler Einnahme. Cmax wird mit Ketamin-Nasenspray zehn bis 17 min nach Applikation erreicht, was zu einem schnelleren Wirkeintritt als bei oraler, sublingualer oder rektaler Administration führt. Der schnelle Wirkeintritt macht nasales Ketamin zu einer Option in der Behandlung von Durchbruchschmerzen, die exakte Dosierung ist allerdings schwer kontrollierbar [3].

\section{Rektale Applikation}

Bei rektaler Applikation werden ähnliche Bioverfügbarkeit und eine ähnliche AUC von Norketamin erreicht wie mit sublingualem Ketamin. Die rektale Verabreichung kann eine Option für Patienten sein, die nicht in der Lage sind zu schlucken [3].

\section{Wirkungsweise von Ketamin im Zentral-Nerven- system}

Die pharmakologische Wirkung von Ketamin beruht auf der nicht kompetitiven Hemmung des N-MethylD-Aspartat Subtyps des Glutamat Rezeptors (NMDA). Damit weist Ketamin unter den Anästhetika und Analgetika einen einzigartigen Wirkmechanismus auf. NMDA-Rezeptoren sind an verschiedenen Abläufen beteiligt, die zur Entstehung chronischer Schmerzen beitragen. Unter anderem spielen sie eine wichtige Rolle im Prozess der zentralen Sensibilisierung [8]. Damit ist Ketamin eine naheliegende Option, um Chronifizierung von Schmerz zu verhindern.

Hinzu kommt jedoch eine Reihe weiterer Effekte an zahlreichen weiteren Rezeptoren. Hierher gehören die tonische Blockierung von spannungsunabhängigen NA-Kanälen (woraus sich eine lokal-anästhetische Wirkung ergibt), die Blockierung von Acetycholin Rezeptoren (bronchodilatatorische Wirkung) sowie in hohen Dosen Opiatrezeptor $(\delta, \mu)$ vermittelte Effekte, die zu einer Potenzierung der Opiatwirkung führen. S-Ketamin zeigt zwei bis dreimal stärkere Affinität zu den Opiatrezeptoren als R-Ketamin. Allerdings beeinflusst Naloxon die Ketamin-Wirkung beim Menschen nicht. Der Anteil der Opiatrezeptor an der Wirkung von Ketamin dürfte also begrenzt sein.

Weiters wirkt Ketamin über den AMPA Rezeptor und metabotrope Glutamat Rezeptoren (mGluR) sowie L-type Ca2+ Kanäle und bewirkt eine Steigerung der Freisetzung von Dopamin und Noradrenalin. Zu diesen Sofortwirkungen kommen verzögerte Effekte, namentlich die Hemmung der IEG (Immediate early genes) Expression (c-fos, c-jun) sowie der Aktivierung von Astrozyten und Mikroglia und damit möglicherweise auch ein Einfluss auf die neuroimmune Interaktion. Ketamin moduliert auch die Phosphorylierung des NMDA Rezeptors [9].

\section{Klinische Wirkung von Ketamin}

Aus diesen komplexen Wirkmechanismen resultieren vielfältige klinische Wirkungen und Einsatzgebiete. Ketamin verbindet Analgesie und rasche Sedierung in einer Substanz, wobei bei niedriger Dosierung die Analgesie im Vordergrund steht [1,3]. Niedrige Dosis ist in der Literatur häufig definiert mit einem Bolus von weniger als $2 \mathrm{mg} / \mathrm{kg}$ intramuskulär oder $1 \mathrm{mg} / \mathrm{kg}$ intravenös [10]. Bezogen auf S-(+)-Ketamin ist als Niedrigdosierung maximal die Hälfte der dieser Werte zu empfehlen.

Niedrig dosiert wirkt Ketamin antihyperalgetisch, antiallodynisch und toleranzprotektiv. Als zusätzliche, potentiell erwünschte Effekte kommen antikonvulsive, antidepressive, antiinflammatorische und neuroprotektive Wirkungen hinzu. Ketamin hat damit ein breites Spektrum an potenziellen Einsatzgebieten. Es beeinträchtigt die hämodynamische Stabilität ebenso wenig wie die Darmmotorik und bewirkt auch in höheren Dosierungen keine Atemdepression.

Damit ergeben sich in der Schmerzmedizin - aber auch darüber hinaus - zahlreiche potenzielle Einsatzgebiete. Ketamin kann präklinisch in der Notfallmedizin eingesetzt werden, sowie intraoperativ und postoperativ zur Prävention chronischer postoperativer Schmerzen. Weitere mögliche Einsatzgebiete sind die Therapie chronischer Schmerzen sowie die Sedoanalgesie auf der Intensivstation, wobei man sich auch den neuroprotektiven Effekt zunutze macht [1, 3].

Während der Einsatz von Ketamin als Monosubstanz bzw. in anästhetisch wirksamer Dosis zur Allgemeinanästhesie wegen unerwünschter psychotroper Wirkungen heute selten geworden ist, sehr wohl aber beim hämodynamisch instabilen Patienten eine Option bleibt, wurden seit den 1990er Jahren zahlreiche randomisierte, kontrollierte Studien zum Einsatz von Ketamin in subanästhetischen Dosierungen in der Behandlung akuter und chronischer Schmerzzustände publiziert. Das neu erwachte Interesse an der Substanz hat auch damit zu tun, dass seit den späten 1990er Jahren das (S)-Enantiomer (S)-Ketamin verfügbar ist, das sich im Vergleich zu (R)-Ketamin durch eine vierfach stärkere analgetische Wirksamkeit auszeichnet. Die Studien zeigen unter anderem, dass Ketamin in analgetischen Dosierungen die unerwünschten Nebenwirkungen hoher Dosen nicht oder nur sehr eingeschränkt zeigt [1-4].

\section{Prävention postoperativer Schmerzen und Opio- id-induzierter Hyperalgesie}

$\mathrm{Zu}$ den gut untersuchten Einsatzgebieten zählt die Prävention von akuten postoperativen Schmerzen durch den Einsatz von niedrig dosiertem Ketamin im perioperativen Schmerzmanagement [11].

Der NMDA-Antagonist Ketamin ist in diesem $\mathrm{Zu}$ sammenhang insofern interessant, als Opioide in 
hohen Dosierungen trotz ihrer starken analgetischen Wirkung die zentrale Sensibilisierung während eines chirurgischen Eingriffs und die daraus resultierende postoperative Hyperalgesie nicht verhindern, sondern sogar verstärken können. Im Tiermodell konnte für niedrig dosiertes (S)-Ketamin ein Effekt nicht nur auf C-Faser-evozierte Potentiale, sondern auch auf die synaptische Langzeit-Potenzierung (eine Form der synaptischen Plastizität) demonstriert werden [12-14].

Auch für den Einsatz beim Menschen gibt es umfangreiche Daten. So konnte zum Beispiel nach großen bauchchirurgischen Eingriffen postoperative Hyperalgesie demonstriert werden, wenn intraoperativ Remifentanil in höheren Dosierungen eingesetzt wurde. Bei Kombination von Remifentanil und Ketamin blieb dieser Effekt ebenso aus, wie bei Verwendung niedriger Remifentanil-Dosen. Damit bietet sich Ketamin als Alternative zu einer Dosissteigerung des Opioids an [15]. Ein systematischer Review randomisierter, kontrollierter Studien fand deutliche Hinweise auf die Reduktion postoperativer Hyperalgesie durch die perioperative Gabe von intravenösem Ketamin, wobei der Effekt unabhängig vom Zeitpunkt der Fentanyl-Gabe, der Art der eingesetzten Opioide und auch der Ketamin-Dosis war [16]. Untersucht wurde der Einsatz von niedrig dosiertem Ketamin auch in Kombination mit epiduraler Analgesie (und Allgemeinanästhesie) bei offenen Laparotomien wegen Rektum-Karzinoms. Dabei zeigte sich im Follow-up bis zu 12 Monaten gute Wirksamkeit, auch in der Vorbeugung einer Schmerzchronifizierung [17].

Detaillierte Empfehlungen für den peri- und intraoperativen Einsatz von (S)-Ketamin wurden 2005 veröffentlicht [18]. Ein relativ rezenter Review zeigt, dass der Einsatz von niedrig dosiertem Ketamin den perioperativen Opioidbedarf um $40 \%$ reduziert, ohne dass dieser Vorteil durch schwere Nebenwirkungen erkauft würde [19]. Eine typische Bolus-Dosis Ketamine beträgt $0,15 \mathrm{mg} / \mathrm{kg}$, die Infusionsrate kann zwischen 0,15 and $1,2 \mathrm{mg} / \mathrm{kg} / \mathrm{h}$ variieren, die Dauer der Behandlung zwischen zwei und $48 \mathrm{~h}$ liegen. Wenn die kumulative Dosis niedrig gehalten wird, sind (insbesondere psychotrope) Nebenwirkungen in der Regel gering $[3,5]$.

Hintergrund der Reduktion des postoperativen Opioid-Bedarfs durch den perioperativen Einsatz von Ketamin dürfte allerdings nicht nur der Einfluss des NDMA-Antagonisten auf die zentrale Sensibilisierung sein. Vielmehr legen Versuche im Tiermodell nahe, dass Ketamin die akute Toleranzentwicklung gegenüber Opioiden verhindert. Zumindest bei der Ratte reichen dafür sehr niedrige Ketamin-Dosen, die allein für sich noch keinen analgetischen Effekt erwarten lassen [20].

Auch antiinflammatorische Effekte (unter anderem eine reduzierte Produktion der Zytokine TNF-alpha und Interleukin 6) dürften an der Prävention postoperativer Hyperalgesie durch Ketamin beteiligt sein [21].
Ein rezentes interdisziplinäres Positionspapier zum perioperativen Schmerzmanagement [11] empfiehlt, Ketamin bei großen Eingriffen als Teil eines multimodalen perioperativen Schmerzmanagements einzusetzen, wenn keine Kontraindikationen vorliegen.

Ketamin hat sich als hilfreich bei der Behandlung Opioid-resistenter akuter postoperativer Schmerzen erwiesen. In einer kontrollierten Studie wurde durch die gemeinsame Gabe einer einzelnen, niedrigen Dosis von Ketamin und Morphin schwerer postoperativer Schmerz substantiell reduziert. Ketamin verbesserte in dieser Studie auch die analgetische Wirkung weiterer Morphin-Gaben [22]. Ein mögliches Erklärungsmodell dafür liefern Versuche im Tiermodell, die zeigen, dass bei Co-Administration von Morphin und Ketamin die Konzentrationen beider Substanzen im Gehirn erhöht werden und Ketamin auch MorphiumToleranz beeinflussen kann [23].

Eine gefürchtete und leider nicht seltene Komplikation verschiedener chirurgischer Eingriffe ist die Chronifizierung postoperativer Schmerzen, von der - je nach Prozedur und Patientenpopulation - bis zu 50\% der Patienten betroffen sind [17, 24].

Eine Prävention chronischer postoperativer Schmerzen ist besonders bei Risikopatienten, also chronischen Schmerzpatienten, Patienten unter Opioidtherapie sowie bei hohem intraoperativen Opioidbedarf anzustreben. Als besondere Risikooperationen gelten Amputationen, Thorakotomien, Mastektomien sowie möglicherweise auch Hernienoperationen [25]. Als weitere Risikofaktoren für persistierende, postoperative Schmerzen gelten weibliches Geschlecht, jüngeres Alter, präoperativer Schmerz, postoperativer Schmerz, verminderte Inhibition bei noxischen Stimuli (conditioned pain modulation, CPM), psychosoziale Prädiktoren, genetische Disposition sowie intraoperative Nervenverletzung.

Ob Ketamin das Risiko der Entwicklung chronisch persistierender postoperativer Schmerzen reduzieren kann, ist in Diskussion. Eine 2016 publizierte Übersichtsarbeit [3] listet Evidenz pro und contra. Einerseits fand einer Metaanalyse substantielle Reduktionen persistierender postoperativer Schmerzen durch perioperatives, intravenöses Ketamin [26], andererseits konnte dies in zwei rezenten randomisierten, kontrollierten Studien für den Post-ThorakotomieSchmerz nicht bestätigt werden [27, 28].

Möglicherweise ist Ketamin in dieser Indikation also eine Option für bestimmte Patientengruppen und bestimmte Eingriffe, die in weiteren Studien identifiziert werden müssen. Ein rezentes österreichisches Positionspapier zum perioperativen Schmerzmanagement betont den Stellenwert von Ketamin im Rahmen von Amputationen [11]. 


\section{Ketamin beim chronischen Schmerz}

\section{Behandlung chronischer Schmerzen}

Eine weitere Besonderheit von Ketamin liegt in seiner Wirksamkeit gegen neuropathischen Schmerz. Diese wird auf die Bedeutung des NMDA Rezeptors in der Genese neuropathischer Schmerzen zurückgeführt [29]. Darüber hinaus gibt es jedoch auch Hinweise, dass Ketamin die absteigende Schmerzhemmung beeinflusst und antiinflammatorische Effekte ausübt. Hinweise auf eine Wirkung von Ketamin in subanästhetischen Dosen auf Mechanismen der absteigenden Schmerzhemmung wurden mittels funktioneller MRT nachgewiesen. Studiendaten legen auch nahe, dass diese Effekte nicht nur bei gesunden Probanden, sondern auch bei Patienten mit small fibre Neuropathie auftreten. Ketamin könnte also dazu beitragen, dass bei Patienten mit neuropathischen Schmerzen die physiologische Schmerz-Inhibition wiederhergestellt wird [30, 31].

Eine kontrollierte Studie verglich die Wirksamkeit von Ketamin in der Behandlung neuropathischer Schmerzen sowohl mit Methadon als auch mit der Kombination von Ketamin und Methadon. Die Ketamin-Monotherapie erwies sich dabei als überlegen im Vergleich zu den beiden anderen Armen, was auf eine bessere Wirkung von Ketamin auf das Symptom Allodynie zurückzuführen war [32].

Die Wirkung von Ketamin bei chronischen Schmerzen, insbesondere wenn diese eine neuropathische Komponente aufweisen, wurde in einer Vielzahl von Studien demonstriert. Allerdings hält ein Review [33] fest, dass die meisten dieser Arbeiten keine praktisch verwertbaren Aussagen liefern, weil sie Ketamin-Infusionen untersuchten und damit oft nur eine auf wenige Stunden begrenzte Wirkung erreichten. In mehreren Studien wurde langanhaltende Schmerzreduktion gesehen, wenn die Ketamin-Infusion über einen Zeitraum von mehreren Tagen ausgedehnt wurde. So konnte beim chronischen, therapieresistenten komplexen regionalen Schmerzsyndrom (CRPS) mit Infusionen analgetischer Ketamin-Dosen über mehrere Tage eine bis zu drei Monate anhaltende Besserung der Schmerzsymptomatik erreicht werden [34]. Der Review betont allerdings, dass zum Einsatz von Ketamin bei chronischem Schmerz keine belastbaren Langzeitdaten vorhanden sind [33]. Ein weiterer Review aus dem Jahr 2016 weist auf die höchst heterogene Datenlage zum Einsatz von Ketamin in der Schmerztherapie hin. So lag in den Studien zum Einsatz von oralem Ketamin die Behandlungsdauer zwischen einer Einmalgabe und einer Dauer-Therapie über 660 Tage. In den meisten Studien wurde zwischen 20 und 80 Tage behandelt. Auch die eingesetzten Dosierungen bewegen sich in einem breiten Spektrum von sehr niedrigen oralen Dosen bis zu $62,5 \mathrm{mg} / \mathrm{kg} / \mathrm{d}$. Die Autoren betonen, dass sie keine Dosis-Wirkungsrelation identifizieren konnten. Während in manchen Arbeiten orales Ketamin in minimalen Dosierungen effektiv war, fanden andere Arbeiten mit hohen intravenösen Dosierungen keine analgetische Wirkung. Sehr wohl wurde jedoch eine Abhängigkeit der Nebenwirkungen von der Dosis gefunden. Die häufig beschriebenen schweren neurologischen und urologischen Komplikationen im Zusammenhang mit chronischem Ketamin-Missbrauch sind erst nach längerem Konsum sehr hoher Dosen zu erwarten, die weit über den gebräuchlichen analgetischen Dosierungen liegen [4].

Daten aus Fallserien weisen eine gute Wirksamkeit von Ketamin bei chronischer, therapierefraktärer Migräne hin. Mittels niedrig dosierter Ketamin-Infusionen wurde bei den Betroffenen über mindestens acht Stunden eine ausgeprägte Besserung der Symptomatik bei guter Verträglichkeit erreicht [35]. Auch hier fehlen noch Langzeitdaten.

Vergleichbare Erfahrungen wurden in einer kürzlich publizierten Fallserie mit Patienten mit refraktärer Migräne oder chronischem Kopfschmerz (new daily persistent headache - NDPH) berichtet. Die 77 Patienten erhielten nach verschiedenen frustranen (auch stationären) Therapieversuchen Infusionen von niedrig dosiertem Ketamin über einen längeren Zeitraum - im Durchschnitt 4,8 Tage. Damit wurde eine signifikante Verbesserung der Symptomatik erreicht. Die Patienten litten bei Aufnahme unter Schmerzen von einer Stärke von 7,1 auf einer Schmerzskala von null bis zehn. Bei Entlassung war der Schmerz auf durchschnittlich 3,8 zurückgegangen $(P<0,0001)$. Von den insgesamt 77 Patienten wurden 55 (71,4\%) als Responder eingestuft. Von diesen 55 Respondern hielt der Effekt bei 15 (27,3\%) auch bis zu einem weiteren Kontrollbesuch an, dieser Vorteil verfehlte jedoch die statistische Signifikanz. Die Autoren betonen, dass Ketamin gut vertragen wurde und fordern kontrollierte Studien [36].

\section{Ketamin und Krebsschmerz}

Der Einsatz von Ketamin bei onkologischen Schmerzpatienten ist besonders dort von Interesse, wo der Schmerz eine deutliche neuropathische Komponente aufweist, und wurde in mehreren offenen Studien untersucht. Dabei wurde bei rund 70 bis $80 \%$ der Patienten gute Schmerzlinderung festgestellt. Bei zehn bis $30 \%$ der Patienten kann jedoch keine suffiziente Analgesie erreicht werden [37].

In einer Fallserie mit jungen Krebspatienten konnte durch den Einsatz von Ketamin als Adjuvans bei acht von elf Patienten eine Reduktion der Opioid-Dosis erreicht werden [38]. Im Gegensatz dazu fand eine randomisierte, placebokontrollierte Studie keinen günstigen Effekt für den Einsatz von subkutanem Ketamin als Adjuvans zur Opioid-Therapie. Ketamin erwies sich in diesem Setting als nicht überlegen im Vergleich zu Placebo, entwickelte dabei jedoch substantielle Nebenwirkungen [39]. Aufgrund dieser Ar- 
beit wurde der Einsatz von Ketamin in der onkologischen Schmerztherapie für einige Jahre weitgehend verlassen. Erst in letzter Zeit regt sich neues Interesse. So gelangte ein 2015 publizierter Review zu dem Ergebnis, dass Ketamin eine interessante Option in der Behandlung von Krebs-Schmerzen darstellt, die nicht mehr auf Opioide ansprechen. Die Autoren betonen allerdings, dass die Evidenz zum Einsatz von Ketamin bei Krebs-Schmerz bei weitem dünner ist als bei nicht-onkologischen Schmerzen. Die meisten der identifizierten Studien waren klein, oftmals methodisch schwach und insgesamt sehr heterogen, fanden jedoch mehrheitlich günstige Effekte durch den Einsatz von Ketamin bei schwer beherrschbarem KrebsSchmerz. Die orale Applikation scheint dabei verträglicher zu sein als die parenterale [40].

Ganz in diesem Sinne empfehlen die Palliative Care Guidelines der European Society for Medical Oncology (ESMO) auf Basis eines systematischen Reviews der Literatur den Einsatz von Ketamin zur palliativen Behandlung von Patienten mit neuropathischen Schmerzen im onkologischen Setting. Diese Behandlung soll unter der Leitung von Spezialisten erfolgen. Empfehlung besteht auch bei komplexen neuropathischen und vaskulären Schmerzsyndromen, bei denen Opioide ihre Wirkung verloren haben. Grundsätzlich sollte Ketamin laut ESMO nur zum Einsatz kommen, wenn Kombinationen aus oralen Opioiden und adjuvanten Analgetika ineffektiv geworden sind. Delir, Anfallsleiden oder Psychose sind Kontraindikationen [41]. Bei erhöhtem intrakraniellem Druck besteht Kontraindikation, wenn der Patient spontanatmend ist.

Wie der Einsatz von Ketamin in der onkologischen Schmerztherapie in der klinischen Praxis aussehen kann, zeigt ein im Wiener Wilhelminenspital eingesetzter Algorithmus [42]. Er kommt bei Patienten mit therapieresistenten Schmerzen trotz Ausschöpfung aller Analgetika-Optionen zum Einsatz und trägt dem individuell sehr unterschiedlichen Ansprechen auf Ketamin sowie dem individuellen Nebenwirkungsprofil Rechnung. Der Patient erhält zunächst eine Probedosis von 5-10 mg S-Ketamin als Kurzinfusion. Wird damit keine Wirkung erzielt, oder treten erhebliche Nebenwirkungen (meist psychotroper Art) auf, so erfolgen keine weiteren Ketamin-Gaben. Bei Respondern, die gute Analgesie bei minimalen Nebenwirkungen erreichen, wird weiter mit Ketamin in intravenöser oder oraler Form behandelt. Auch der Einsatz von Ketamin in der Schmerzpumpe ist eine Option, wobei die Hoffnung besteht, dass die frühe Kombination von Opioiden mit Ketamin Dosiseskalationen eine Toleranzentwicklung verhindern kann. Kandidaten sind Patienten mit einem Morphinbedarf (Pumpe oder i.v.) von mehr als $200 \mathrm{mg}$ pro Tag.

Der Versuch, chemotherapieinduzierte, periphere Neuropathie mit topischem Ketamin/Amitriptylin zu behandeln, erwies sich in einer kontrollierten Studie als wirkungslos [43].

\section{Ketamin in der Behandlung von Depressionen}

Niedrig dosiertes Ketamin wird gegenwärtig auch als neuartiges, schnell wirkendes Antidepressivum beforscht und diskutiert. Die Literatur enthält Nachweise für eine rasche antidepressive Wirkung von niedrig dosiertem intravenösen Ketamin bei „Major“-Depressionen, bei bipolarer Depression und bei Depression mit Suizidgedanken. Die vermuteten Wirkmechanismen von Ketamin unterscheiden sich deutlich von jenen anderer antidepressiv wirksamer Substanzen und gehen auch über die bekannte Ketamin-Wirkung auf den NMDAR hinaus. So wurde Ketamin im Tiermodell mit der mTOR- (mammalian target of rapamycin-) abhängigen Synapsenbildung im präfrontalen Kortex der Ratte, der eukaryotischen Elongationsfaktor 2- (peEF2-) Phosphorylierung und der Glykogen-Synthase-Kinase (GSK-3) in Verbindung gebracht. Auch in dieser Indikation scheint der limitierende Faktor die transiente Natur der antidepressiven Wirkung von Ketamin zu sein. Darüber hinaus bestehen Bedenken in Bezug auf Missbrauch und Abhängigkeit [44].

Wie deutlich die antidepressive Wirkung sein kann, zeigte beispielsweise eine randomisierte, kontrollierte Studie mit einer einzigen Infusion von Ketamin im Vergleich zu einer aktiven Placebo-Kontrolle mit dem Anästhetikum Midazolam. Eingeschlossen waren Patienten mit behandlungsresistenter Depression während einer depressiven Phase. Ketamin zeigte in diesem Setting eine schnelle antidepressive Wirkung bei guter Verträglichkeit. Dissoziative Symptome traten bei $17 \%$ der Patienten auf, nach sieben Tagen bestand diesbezüglich jedoch kein Unterschied mehr zwischen den Gruppen [45]. Auch hier fehlen Informationen zu Response-Dauer und Sicherheit, die vor der Umsetzung in die klinische Praxis erforderlich wären. In Fallberichten wurde auch die Wirksamkeit von oralem Ketamin bei der therapieresistenten Depression dokumentiert [46]. Ebenfalls vielversprechende Ergebnisse brachte eine offene Pilotstudie, die den Einfluss von täglichem oralem Ketamin auf Angst und Depression von 14 Hospiz-Patienten untersuchte und dabei über vier Wochen ausgezeichnetes Ansprechen fand [47].

Von besonderem Interesse ist eine in Studien dokumentierte Wirkung von Ketamin auf das für die Patienten besonders belastende und durch herkömmliche medikamentöse Therapien kaum beeinflussbare Symptom der Anhedonie. Eine randomisierte, placebokontrollierte Studie mit 36 Patienten mit therapieresistenter bipolarer Depression konnte zeigen, dass eine einzelne Ketamin-Infusion das Anhedonie-Niveau signifikant über die gesamte Beobachtungsdauer von 14 Tagen reduzieren kann [48].

In einer offenen Studie erreichte die gleiche Gruppe mit einer Ketamin-Infusion auch bei Patienten mit unipolarer Depression eine deutliche Reduktion der Anhedonie. Es konnte weiters gezeigt werden, dass die Besserung der Anhedonie mit verstärktem Glukose- 
Metabolismus im Hippocampus und im dorsalen anterioren Gyrus cinguli (dACC) sowie reduziertem Metabolismus im Gyrus frontalis inferior, sowie dem orbitofrontalen Cortex (OFC) einhergeht [49].

Mehrere neuere Reviews erlauben eine bessere Einordnung des möglichen Stellenwerts von Ketamin in der Behandlung uni- und bipolarer Depressionen. Diese Arbeiten zeigen allerdings auch, wie begrenzt die Datenlage zum gegenwärtigen Zeitpunkt noch ist. Ein Review auf Basis einer Datensuche auf EMBASE, PsycINFO, CENTRAL und Medline für die Zeit von 1962 bis Januar 2014 fand sieben randomisierte kontrollierte Studien zum Einsatz von intravenösem und eine für nasales Ketamin in der Behandlung schwerer Depression (major depressive episodes). Insgesamt waren in diese Studien 73 Patienten in Parallelgruppen- und 110 Patienten in Crossover-Studien eingeschlossen. Dabei erwies sich Ketamin zu allen untersuchten Zeitpunkten ( $24 \mathrm{~h}$ bis sieben Tage) gegenüber dem Vergleichsarm als überlegen. Die Wirksamkeit war bei unipolarer Depression besser als bei bipolarer. Ketamin erwies sich als assoziiert mit vorübergehenden psychotomimetischen Effekten, führte jedoch zu keiner anhaltenden Psychose oder einem affektiven Switch [50]. Ein weiterer Review unterstreicht den zunehmenden off-label Einsatz von Ketamin in psychiatrischen Indikationen. So werden KetaminInfusionen bei refraktärer Depression zunehmend als Ersatz für die Elektro-Krampftherapie (EKT) eingesetzt. Die Kombination von Ketamin und EKT hat sich hingegen als unverträglich und unwirksam erwiesen [51]. Ungeachtet der dokumentierten Erfolge bleiben noch zahlreiche offene Fragen. Dies betrifft insbesondere die Dosierung sowie Strategien, die eine langfristige antidepressive Behandlung mit Ketamin möglich machen [10, 52].

In dieser Situation hat die American Psychiatric Association Council of Research Task Force on Novel Biomarkers and Treatments kürzlich ein KonsensusStatement zum Einsatz von Ketamin in der Psychiatrie herausgegeben [53]. Die Autoren betonen, dass dieser Einsatz grundsätzlich off label erfolgt und die dahinterstehende Evidenz sehr begrenzt ist. Generell sei es nur möglich Empfehlungen zur Behandlung schwerer Depressionen ohne psychotische Symptome zu geben, da für alle anderen Indikationen die Datenlage gegenwärtig zu dünn ist. Auch in diesem Dokument wird darauf hingewiesen, dass die verfügbaren Daten überwiegend aus Studien mit maximal einer Woche Beobachtungsdauer nach einer Einzelinfusion von Ketamin stammen und nur in wenigen Arbeiten die Effekte wiederholter Ketamin-Gabe untersucht wurden $[54,55]$. Solche Regime würden zwar in mehreren Zentren angewandt, es gäbe jedoch so wenig publizierte Resultate, dass keine evidenzbasierten Empfehlungen gegeben werden können.

Der Konsensus hält fest, dass Patienten vor einer Behandlung mit Ketamin sorgfältig abgeklärt und informiert werden müssen. Informed consent muss die
Grundlage der Behandlung bilden. Diese ist an einem Zentrum durchzuführen, in dem die Möglichkeiten für kardiovaskuläres Monitoring bzw. die erforderlichen Interventionen im Falle von Komplikationen gegeben sind. Das Zentrum muss auch auf die Möglichkeit einer psychotischen Reaktion seitens des Patienten vorbereitet sein. Hinsichtlich Dosierung und Applikationsweg ist die Studienlage heterogen, am besten untersucht ist die intravenöse Infusion von $0,5 \mathrm{mg} / \mathrm{kg}$ über $40 \mathrm{~min}$.

\section{Missbrauchspotential und Nebenwirkungen}

Hinsichtlich der Nebenwirkungen bereiten vor allem die psychotropen Effekte von Ketamin Sorgen - insbesondere bei längerfristiger Einnahme. Bei gesunden Menschen kann eine akute Dosis des N-Methyl-DAspartat- (NMDA-) Rezeptor-Antagonisten Ketamin deutliche Psychose-ähnliche Effekte und kognitive Beeinträchtigungen induzieren. Ketamin hat auch Missbrauchspotential.

Ketamin-Nebenwirkungen sind in der Regel dosisabhängig und spontan reversibel [56]. Hierbei ist zu beachten, dass Ketamin für Zwecke der Therapie chronischer Schmerzen oder Depressionen in Dosierungen angewendet wird, die deutlich unter der anästhetischen Dosierung liegen. Die therapeutische Breite macht es zu einem sicheren Arzneimittel [26].

$\mathrm{Zu}$ den Nebenwirkungen von Ketamin, speziell in anästhetischen Dosierungen, gehören eine Zunahme der Herzfrequenz und des arteriellen Blutdrucks sowie eine Steigerung des myokardialen Sauerstoffverbrauchs. Aufgrund dieser Effekte sind arterielle Hypertonie, Herzerkrankungen wie chronische Herzinsuffizienz, Herzfehler oder koronare Herzerkrankung absolute Kontraindikationen für den Ketamin-Einsatz. Ebenso sprechen schlecht eingestellte Hyperthyreose oder Aneurysmen gegen einen Ketamin-Einsatz. Gegen die diskutierten Bedenken, dass Ketmin den Hirndruck erhöhen könnte, sprechen rezentere Studien [57]. Glaukom, psychische Erkrankungen oder Epilepsie in der Anamnese, Porphyrie oder die Anwendung im ersten Trimester der Schwangerschaft stellen Kontraindikationen dar [58-60].

Die atemdepressive Wirkung von Ketamin ist nur gering, die relaxierende Wirkung auf die Bronchialmuskulatur kann einen Asthmaanfall durchbrechen. Der intraokuläre Druck wird durch Ketamin gesteigert, ebenso der Tonus der Uterusmuskulatur. Hypersalivation und eine gesteigerte Bronchialsekretion gehören zu weiteren Nebenwirkungen von Ketamin [Fachinformation]. Durch die zusätzliche Verwendung von Sedativa zur Reduzierung der psychodelischen Nebenwirkungen wird das Risiko einer Atemdepression erhöht.

Chronischer Ketamingebrauch kann zu Schädigungen im Urogenitaltrakt führen, der bei Abstinenz aber wieder reversibel ist. Dazu gehören Schmerzen im Bereich der Harnblase, Inkontinenz oder Papillennekrosen [60]. 
Was die psychoaktiven Eigenschaften von Ketamin betrifft, kann es auch in subanästhetischer Dosierung $\mathrm{zu}$ dissoziativen Symptomen, Halluzination, Dysphorie, Angst oder Orientierungsstörungen kommen [Fachinformation].

Angesichts der zentralen Wirksamkeit von Ketamin ist mit anderen psychoaktiven Substanzen wie Alkohol, Opioiden, Benzidiazepinen sowie Cannabis und Cannabinoiden Vorsicht angezeigt [56].

Die durchaus verbreitete „Freizeit-Einnahme“ von Ketamin unter jungen Menschen liefert einige Daten zu langfristigen Nebenwirkungen. Im Zusammenhang mit chronischem Ketamin-Konsum wird eine Vielzahl von psychiatrischen und neurologischen Komplikationen beschrieben. Todesfälle durch Überdosierung sind selten und stehen in der Regel in Zusammenhang mit der Einnahme anderer psychoaktiver und/ oder sedierender Substanzen wie zum Beispiel Alkohol. Injektionen von Ketamin-Dosen weit über den in der Anästhesie üblichen Dosierungen wurden ohne Folgeschäden überlebt [61, 62].

So wurden in einer epidemiologischen Studie häufige Ketamin-User, seltene Ketamin-User, abstinente User, polytoxikomane Kontrollen und Nicht-Nutzer von illegalen Drogen über ein Jahr verglichen. Die Studie fand in Gruppe der häufigen Nutzer kognitive Defizite und zunehmenden Ketaminkonsum in Verbindung mit abnehmender Leistung hinsichtlich des räumlichen Arbeitsgedächtnisses sowie eine Verschlechterung in Mustererkennungs-Gedächtnisaufgaben. Die Bewertung des psychischen Wohlbefindens zeigte mehr dissoziative Symptome bei „Vielnutzern" und einen Dosis-Wirkungs-Effekt auf wahnhafte Symptome, mit höheren Scores bei „Vielnutzern“ als bei seltenen, abstinenten Usern und Nicht-Usern. Sowohl „Vieluser“ als auch die abstinente Gruppe zeigten erhöhte Depressionswerte über die 12 Monate [63]. Aus diesen Daten lässt sich ableiten, dass eine starke Nutzung von Ketamin sowohl hinsichtlich der kognitiven Funktion als auch des psychischen Wohlbefindens nachteilige Wirkungen hat. Wie weit dies auch beim medizinisch indizierten Einsatz von Ketamin zutrifft, bzw. wie weit sich Missbrauchspotential und psychotrope Nebenwirkungen von Ketamin beherrschen lassen, müssen Studien zeigen.

\section{Einhaltung ethischer Richtlinien}

Interessenkonflikt W. Jaksch, R. Likar und M. Aigner geben an, dass kein Interessenkonflikt besteht.

Ethische Standards Für diesen Beitrag wurden von den $\mathrm{Au}$ toren keine Studien an Menschen oder Tieren durchgeführt.

Open Access Dieser Artikel wird unter der Creative Commons Namensnennung 4.0 International Lizenz (http:// creativecommons.org/licenses/by/4.0/deed.de) veröffentlicht, welche die Nutzung, Vervielfältigung, Bearbeitung, Verbreitung und Wiedergabe in jeglichem Medium und Format erlaubt, sofern Sie den/die ursprünglichen Autor(en) und die Quelle ordnungsgemäß nennen, einen Link zur Creative
Commons Lizenz beifügen und angeben, ob Änderungen vorgenommen wurden.

\section{Literatur}

1. Annetta MG, Iemma D, Garisto C, et al. Ketamine: new indications for an old drug. Curr Drug Targets. 2005;6:789-94.

2. Li L, Vlisides PE. Ketamine:50 years of modulating the mind. Front Hum Neurosci. 2016;10:612.

3. Peltoniemi MA, Hagelberg NM, Olkkola KT, et al. Ketamine: a review of clinical pharmacokinetics and pharmacodynamics in anesthesia and pain therapy. Clin Pharmacokinet. 2016;55(9):1059-77.

4. Schoevers RA, Chaves TV, Balukova SM, et al. Oral ketamine for the treatment of pain and treatment-resistant depression. BrJPsychiatry. 2016;208(2):108-13.

5. Fanta S, Kinnunen M, Backman JT, et al. Population pharmacokinetics of S-ketamine and norketamine in healthy volunteers after intravenous and oral dosing. Eur J Clin Pharmacol.2015;71:441-7.

6. Chong C, Schug SA, Page-Sharp M, et al. Development of a sublingual/oral formulation of ketamine for use in neuropathicpain:preliminaryfindingsfromathree-wayrandomized, crossover study. Clin Drug Investig. 2009;29:217-24.

7. Christensen K, Rogers E, Green GA, et al. Safety and efficacy of intranasal ketamine for acute postoperative pain. Acute Pain. 2007;9:183-92.

8. Pelissier T, Laurido C, Kramer V, et al. Antinociceptive interactions of ketamine with morphine or methadone in mononeuro-pathic rats. Eur J Pharmacol. 2003;477:23-8.

9. Sleigh J, Harvey M, Voss L, et al. Ketamine-More mechanisms of action than just NMDA blockade. Trends Anaesth CritCare. 2014;4:76-81.

10. Salvadore G, Singh JB. Ketamine as a fast acting antidepressant: current knowledge and open questions. CNS Neurosci Ther. 2013;19(6):428-36.

11. Likar R, Jaksch W, Aigmüller T, et al. Interdisziplinäres Positionspapier „Perioperatives Schmerzmanagement“. Schmerz. 2017;31(5):463-82.

12. BenrathJ,BrechtelC,StarkJ, etal.Lowdose ofS(+)-ketamine prevents long-term potentiation in pain pathways under strong opioid analgesia in the rat spinal cord in vivo. Br J Anaesth. 2005;95:518-23.

13. Guignard B, Bossard AE, Coste C, et al. Acute opioid tolerance: intraoperative remifentanil increases postoperative pain and morphine requirement. Anesthesiology. 2000;93:409-17.

14. Liu XG, Sandkühler J. Long-term potentiation of C-fiberevoked potentials in the rat spinal dorsal horn is prevented by spinal N-methyl-D-aspartic acid receptor blockage. Neurosci Lett. 1995;191:43-6.

15. Joly V, Richebe P, Guignard B, et al. Remifentanil-induced postoperative hyperalgesia and its prevention with smalldose ketamine. Anesthesiology. 2005;103(1):147-55.

16. Laskowski K, Stirling A, McKayWP, et al. A systematic review of intravenous ketamine for postoperative analgesia. Can J Anaesth. 2011;58(10):911-23.

17. Lavand'homme P, De Kock M, Waterloos H. Intraoperative epidural analgesia combined with ketamine provides effective preventive analgesia in patients undergoing major digestive surgery. Anesthesiology. 2005;103(4):813-20.

18. Himmelseher S, Durieux ME. Ketamine for perioperative pain management. Anesthesiology. 2005;102(1):211-20.

19. Jouguelet-Lacoste J, La Colla L, Schilling D, et al. The use of intravenous infusion or single dose of low-dose ketamineforpostoperativeanalgesia:areview of the current literature. Pain Med.2015;16:383-403. 
20. Kissin I, Bright CA, Bradley EL Jr.. The effect of ketamine on opioid-induced acute tolerance: can it explain reduction of opioid consumption with ketamine-opioid analgesic combinations? Anesth Analg. 2000;91:1483-8.

21. Kurdi MS, Theerth KA, Deva RS. Ketamine: current applications in anesthesia, pain, and critical care. Anesth Essays Res. 2014;8(3):283-90.

22. Weinbroum AA. A single small dose of postoperative ketamine provides rapid and sustained improvement in morphine analgesiain the presence of morphine-resistant pain. Anesth Analg. 2003;96:789-95.

23. Lilius TO, Jokinen V, Neuvonen MS, et al. Ketamine coadministration attenuates morphine tolerance and leads to increased brain concentrations of both drugs in the rat. Br J Pharmacol.2015;172(11):2799-813.

24. Kehlet H, Jensen TS, Woolf CJ. Persistent postsurgical pain: riskfactors and prevention. Lancet. 2006;367:1618-25.

25. Schug SA, Pogatzki-Zahn EM. Chronic pain after surgery or injury. Pain 19(1):1-5.

26. McNicol ED, Schumann R, Haroutounian S. A systematic review and meta-analysis of ketamine for the prevention of persistent post-surgical pain. Acta Anaesthesiol Scand. 2014;58:1199-213.

27. Hu J, Liao Q, Zhang F, et al. Chronic postthoracotomy pain and perioperative ketamine infusion. J Pain Palliat Care Pharmacother. 2014;28:117-21.

28. Tena B, Gomar C, Rios J. Perioperative epidural or intravenous ketamine does not improve the effectiveness of thoracic epidural analgesia for acute and chronic pain after thoracotomy. Clin J Pain. 2014;30:490-500.

29. Petrenko AB, Yamakura T, Baba H, et al. The role of N-methyl-D-aspartate (NMDA) receptors in pain: a review. Anesth Analg. 2003;97:1108-16.

30. Niesters M, Khalili-Mahani N, Martini C, et al. Effect of subanesthetic ketamine on intrinsic functional brain connectivity: a placebo controlled functional magnetic resonance imaging study in healthy volunteers. Anesthesiology. 2012;117:868-77.

31. Niesters M, Aarts L, Sarton E, et al. Influence of ketamine and morphine on descending pain modulation in chronic pain patients: a randomized placebo controlled cross-over study. BrJAnaesth. 2013;110(6):1010-6.

32. Rigo FK, Trevisan G, Godoy MC, et al. Management of neuropathic chronic pain with methadone combined with ketamine: a randomized, double blind, active-controlled clinical trial. Pain Physician. 2017;20:207-15.

33. Niesters M, Martini C, Dahan A. Ketamine for chronic pain: risks and benefits. Br J Clin Pharmacol. 2014;77(2): 357-67.

34. Sigtermans MJ, van Hilten JJ, Bauer MC, et al. Ketamine produces effective and long-term pain relief in patients with complex regional pain syndrome type 1. Pain. 2009;145:304-11.

35. Lauritsen C, Mazuera S, Lipton RB, et al. Intravenous ketamine for subacute treatment of refractory chronic migraine: a case series. JHeadache Pain. 2016;17:106.

36. Pomeroy JL, Marmura MJ, Nahas SJ, et al. Ketamine infusions for treatment refractory headache. Headache. 2017;57(2):276-82.

37. Zech DF, Grond S, Lynch J, et al. Validation of world health organization guidelines for cancer pain relief: a 10-year prospective study. Pain. 1995;63(1):65-76.

38. Finkel JC, Pestieau SR, Quezado ZMN. Ketamine as an adjuvant for treatment of cancer pain in children and adolescents. J Pain. 2007;8(6):515-21.

39. Hardy J, Quinn S, Fazekas B, et al. Randomized, doubleblind, placebo-controlled study to assess the efficacy and toxicity of subcutaneous ketamine in the management of cancer pain. J Clin Oncol.2012;30(29):3611-7.

40. Zgaia AO, Irimie A, Sandesc D, et al. The role of ketamine in the treatment of chronic cancer pain. Clujul Med. 2015;88(4):457-61.

41. Ripamonti CI, Santini D, Maranzano E, et al. ESMO Guidelines Working Group. Management of cancer pain: ESMO Clinical Practice Guidelines. Ann Oncol. 2012;23(Suppl 7):vii139-vii54.

42. Bell RF, Jaksch W, Kalso EA. Interpreting the evidence: reply to Spruyt et al. J Pain Symptom Manage. 2014;47(4):e2-e4.

43. Gewandter JS, Mohile SG, Heckler CE, et al. A phase III randomized, placebo-controlled study of topical amitriptyline and ketamine for chemotherapy-induced peripheral neuropathy (CIPN): a university of rochester CCOP study of 462 cancer survivors. Support Care Cancer. 2014;22(7): 1807-14.

44. Naughton M, Clarke G, O'Leary OF, et al. A review of ketamine in affective disorders: current evidence of clinical efficacy, limitations of use and pre-clinical evidence on proposed mechanisms of action. JAffect Disord. 2014;156:24-35.

45. Murrough JW, Iosifescu DV, Chang LC, et al. Antidepressant efficacy of ketamine in treatment-resistant major depression: atwo-siterandomized controlled trial.AmJPsychiatry. 2013;170:1134-42.

46. Swiatek KM, Jordan K, Coffman J. New use for an old drug: oralketamine for treatment-resistant depression. BMJCase Rep. 2016;3:2016.

47. Irwin SA, Iglewicz A, Nelesen RA, et al. Daily oral Ketamine for the treatment of depression and anxiety in patients receiving hospice care: a 28-day open-label proof-of-concept trial. J Palliat Med. 2013;16(8):958-65.

48. Lally N, Nugent AC, Luckenbaugh DA, et al. Anti-anhedonic effect of ketamine and its neural correlates in treatmentresistantbipolardepression. TranslPsychiatry. 2014;4:e469.

49. Lally N, Nugent AC, Luckenbaugh DA, et al. Neural correlates of change in major depressive disorder anhedonia following open-label ketamine. J Psychopharmacol. 2015;29(5):596-607.

50. McGirr A, Berlim MT, Bond DJ, et al. A systematic review and meta-analysis of randomized, double-blind, placebocontrolled trials of ketamine in the rapid treatment of major depressive episodes. Psychol Med. 2015;45(4):693-704.

51. Bobo WV, Vande Voort JL, Croarkin PE, et al. Ketamine for treatment-resistant unipolar and bipolar major depression: critical review and implications for clinical practice. Depress Anxiety. 2016;33(8):698-710.

52. Wilkinson ST, Sanacora G. Considerations on the off-label use of ketamine as a treatment for mood disorders. JAMA. 2017;318(9):793-4.

53. Sanacora G, Frye MA, McDonald W, et al. A consensus statement on the use of ketamine in the treatment of mood disorders. JAMA Psychiatry. 2017;74(4):399-405.

54. Murrough JW, Perez AM, Pillemer S, et al. Rapid and longerterm antidepressant effects of repeated ketamine infusions in treatment-resistant major depression. Biol Psychiatry. 2013;74(4):250-6.

55. Singh, et al. A double-blind, randomized, placebo-controlled, dose-frequency study of intravenous ketamine in patients with treatment-resistant depression. Am J Psychiatry. 2016;173(8):816-26.

56. Fachinformation Ketanest ${ }^{\circledR}$, Stand 06 (2017)

57. Wang X, DingX, Tong Y, et al. Ketamine does not increase intracranial pressure compared with opioids: meta-analysis of randomized controlled trials. J Anesth. 2014;28(6):821-7.

58. Larsen R. Anästhesie. München, Jena: Elsevier, Urban \& Fischer; 2010 . 


\section{übersicht}

59. Sinner B, Graf BM. Ketamine. Handb Exp Pharmacol. 2008;182:313-33.

60. Thiel H, Roewer N. Anästhesiologische Pharmakotherapie: von den Grundlagen der Pharmakologie zur Medikamentenpraxis. Stuttgart, New York: Thieme; 2009.

61. Tsai TH, Cha TL, Lin TT, et al. Ketamin-associated bladder dysfunction. IntJ Urol. 2009;16:826-9.

62. Wood D, Cottrell A, Baker SC, Southgate J, et al. Recreational ketamine: from pleasure to pain. BJU Int. 2011;107(12):1881-4.
63. Jansen KLR. A review of the nonmedical use of ketamine: use, users and consequences. J Psychoactive Drugs. 2000;32(4):419-33.

Hinweis des Verlags Der Verlag bleibt in Hinblick auf geografische Zuordnungen und Gebietsbezeichnungen in veröffentlichten Karten und Institutsadressen neutral. 